\title{
Study of the Start Point of Optimization Trajectories for Complex Strategies of the Circuit Design Process
}

\author{
ALEXANDER ZEMLIAK \\ Department of Physics and Mathematics \\ Autonomous University of Puebla \\ Av. San Claudio s/n, Puebla, 72570 \\ MEXICO
}

\begin{abstract}
The different design trajectories have been analyzed in the design space on the basis of the new system design methodology. Optimal position of the design algorithm start point was analyzed to minimize the CPU time. The initial point selection has been done on the basis of the before discovered acceleration effect of the system design process. The geometrical dividing surface was defined and analyzed to obtain the optimal position of the algorithm start point. The numerical results of the design of passive and active nonlinear electronic circuits confirm the possibility of the optimal selection of the starting point of the design algorithm.
\end{abstract}

Key-Words: - Time-optimal design algorithm, control theory application, optimal start point selection

Received: January 8, 2021. Revised: June 14, 2021. Accepted: July 1, 2021. Published: July 12, 2021.

\section{Introduction}

The problem of the computer time reduction of a large system design is one of the essential problems of the total quality design improvement. Besides the traditionally used ideas of sparse matrix techniques and decomposition techniques [1]-[4] some another ways were determine to reduce the total computer design time. The techniques for analog circuit optimization can be classified in two main groups: deterministic optimization algorithms and stochastic search algorithms. Practical methods of optimization were developed for circuit designing, timing, and area optimization [5-6]. However, classical deterministic optimization algorithms may have a number of drawbacks: they may require that a good initial point be selected in the parameter space, they may reach an unsatisfactory local minimum, and they require that the cost function be continuous and differentiable. To overcome these issues, special methods were applied to determine the initial point of the process by centering [7] or applying geometric programming methods [8].

Stochastic search algorithms, especially evolutionary computation algorithms like genetic algorithms, differential evaluation, genetic programming, particle swarm optimization, etc. have been developed in recent years [9-15]. Genetic algorithms have been employed as optimization routines for analog circuits due to the ability to find a satisfactory solution. A special algorithm defined as a particle swarm optimization technique is one of the evolutionary algorithms and competes with genetic algorithms. This method is successfully used for electromagnetic problems and for optimization of microwave systems [16-17].

A more general formulation of the circuit optimization problem was developed on a heuristic level some decades ago [18]. This approach ignored Kirchhoff's laws for all or part of a circuit during the optimization process. The practical aspects of this idea were developed for the optimization of microwave circuits [19] and for the synthesis of high-performance analog circuits [20] in an extreme case where all the equations of the circuit were not solved during the optimization process.

In work [21] the problem of circuit optimization is formulated in terms of the theory of optimal control. Thus, the process of circuit optimization was generalized and defined as the dynamic controllable system. In this case, the basic element is the control vector that changes the structure of the equations of the system of optimization process. Thus, there is a set of strategies of optimization that have different number of operations and different computing times.

This approach serves for the time-optimal design algorithm definition. On the other hand this approach gives the possibility to analyze with a great clearness the design process while moving along the trajectory curve in the design space. The main conception of this approach is the introduction of the special control functions, which, on the one hand generalize the design process and, on the other hand, they give the possibility to control design 
process to achieve the optimum of the design objective function in the minimum computer time. This possibility appears because practically an infinite number of the different design strategies that exist within the bounds of the theory, but the different design strategies have the different operation number and executed computer time. On the bounds of this conception, the traditional design strategy is only a one representative of the enormous set of different design strategies. As shown in [21] the potential computer time gain that can be obtained by the new design problem formulation increases when the size and complexity of the system increase but it is realized only in case when we have the algorithm for the optimal trajectories real construction. We can define the formulation of the intrinsic properties and special restrictions of the optimal design trajectory as one of the first problems that needs to be solved for the optimal algorithm construction.

\section{Problem Formulation}

The design process for any analog system design can be defined [21] as the problem of the generalized objective function $F(X, U)$ minimization by means of the vector equation:

$$
X^{s+1}=X^{s}+t_{s} \cdot H^{s}
$$

with the constraints:

$$
\left(1-u_{j}\right) g_{j}(X)=0, j=1,2, \ldots, M
$$

where $X \in R^{N}, X=\left(X^{\prime}, X^{\prime \prime}\right), X^{\prime} \in R^{K}$ is the vector of the independent variables and the vector $X^{\prime \prime} \in R^{M}$ is the vector of dependent variables $(N=K+M), g_{j}(X)$ for all $j$ is the system model, $s$ is the iterations number, $t_{s}$ is the iteration parameter, $t_{s} \in R^{1}, H \equiv H(X, U)$ is the direction of the generalized objective function $F(X, U)$ decreasing, $U$ is the vector of the special control functions $U=\left(u_{1}, u_{2}, \ldots, u_{M}\right)$, where $u_{j} \in \Omega ; \Omega=\{0 ; 1\}$. The generalized objective function $F(X, U)$ is defined as: $F(X, U)=C(X)+\psi(X, U)$ where $C(X)$ is the ordinary cost function of the design process, and $\psi(X, U)$ is the additional penalty function: $\psi(X, U)=\frac{1}{\varepsilon} \sum_{j=1}^{M} u_{j} \cdot g_{j}^{2}(X)$. This problem formulation permits to redistribute the computer time expense between the problem (2) solve and the optimization procedure (1) for the function $F(X, U)$. The control vector $U$ is the main tool for the redistribution process in this case. Practically an infinite number of the different design strategies are produced because the vector $U$ depends on the optimization current step. We can define two special strategies: Traditional Design Strategy (TDS) for $U=(0,0, \ldots, 0)$ and Modified Traditional Design Strategy (MTDS) for $U=(1,1, \ldots, 1)$. The problem of the optimal design strategy search is formulated now as the typical problem for the functional minimization of the control theory. The functional that needs to minimize is the total CPU time $T$ of the design process. This functional depends directly on the operations number and more generally on the design trajectory that has been realized. The main difficulty of this problem definition is unknown optimal dependencies of all control functions $u_{j}$. This problem is the central for such a type of the design process definition.

\section{Trajectory Analysis}

The problem of the initial point selection for the design process is one of the essential problems of the time-optimal algorithm construction. The analysis of the acceleration effect for the simplest electronic circuit shown in Fig.1 was carried out in [22]. This is the two-dimensional case.

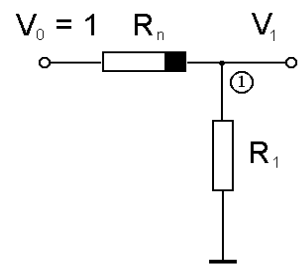

Fig.1. Simplest one node circuit.

The vector of the state variables $X$ has two components $X=\left(x_{1}, x_{2}\right)$ where $x_{1}^{2}=R_{1}, x_{2}=V_{1}$. The nonlinear element has the following dependency: $R_{n}=r_{0}+b V_{1}$. Using the Laws of Kirchhoff we can obtain the following function $g(X)$ :

$$
g(X) \equiv\left(x_{1}^{2}+r_{0}+b x_{2}\right) x_{2}-x_{1}^{2}=0
$$

The objective function is defined by the formula $C(X)=\left(x_{2}-k_{V}\right)^{2}$, where $k_{V}$ has the fixed value. There is only one control function $u_{1}$ in this case because there is only one dependent parameter $x_{2}$. The design trajectory for this example is the curve 
in two-dimensional space, if the numerical design algorithm is applied.

The optimization procedure and the electronic system model, in accordance with the new design methodology [21], are defined by the next two equations:

$$
\begin{aligned}
& x_{i}^{s+1}=x_{i}^{s}+t_{s} \cdot f_{i}(X, U), i=1,2 \\
& \left(1-u_{1}\right) g(X)=0
\end{aligned}
$$

where $U$ is the vector of control variables, and the components of the movement directions $f_{i}(X, U)$ for the $i=1,2$ depend on the optimization method. These functions, for the gradient method for example, are given by the formulas:

$$
\begin{aligned}
& f_{1}(X, U)=-\frac{\delta}{\delta x_{1}} F(X, U) \\
& f_{2}(X, U)=-u_{1} \frac{\delta}{\delta x_{2}} F(X, U)+\frac{\left(1-u_{1}\right)}{t_{s}}\left[-x_{2}^{s}+\eta_{2}(X)\right]
\end{aligned}
$$

where $F(X, U)$ is the generalized objective function, $F(X, U)=C(X)+\frac{1}{\varepsilon} u_{1} g_{1}^{2}(X), \eta_{2}(X)$ is the implicit function $\left(x_{2}^{s+1}=\eta_{2}(X)\right)$ and it gives the value of the parameter $x_{2}$ from the equation (5), and the operator $\frac{\delta}{\delta x_{i}}$ for $i=1,2$ means: $\frac{\delta}{\delta x_{1}} F=\frac{\partial F}{\partial x_{1}}+\frac{\partial F}{\partial x_{2}} \frac{\partial x_{2}}{\partial x_{1}}, \frac{\delta}{\delta x_{2}} F=\frac{\partial F}{\partial x_{2}}$.

As shown in [21] we need to select the initial point of the design process with the negative coordinate $x_{2}$. In this case the acceleration process can be realized. The family of the design curves for the circuit on Fig. 1, which corresponds to the modified traditional design strategy $(\mathrm{u}=1)$ and the negative initial value of the second coordinate $\left(x_{2}<0\right)$ of the vector $\mathrm{X}$ is shown in Fig. 2 for the 2-D phase space. These curves have different start points but the same final point $\mathrm{F}$. The start points were selected on the circle arc and have the different initial coordinates. The special curve S-F, which is marked by thick line, is the separating curve. This curve separates the trajectories that are the candidates for the acceleration effect achievement (all curves that lie under the curve $S$ $\mathrm{F})$, and the trajectories that can not produce the acceleration effect (curves that lie over the curve SF). It is clear that the projections of the final point $F$ to all curves of the first group define the switching point of the optimal trajectory, which produces the acceleration effect. All curves of the first group (17) approach to the final point $F$ from the left side, and all curves of the second group (9-16) approach to the final point from the right side. The comparison of the relative computer time for all curves of the Fig. 2 is shown in Fig. 3.

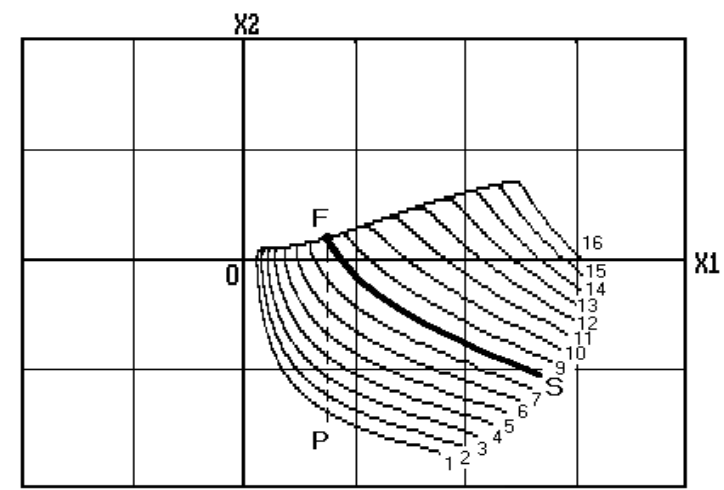

Fig.2. Trajectories of the MTDS for the different start points with the negative coordinate $x_{2}$.

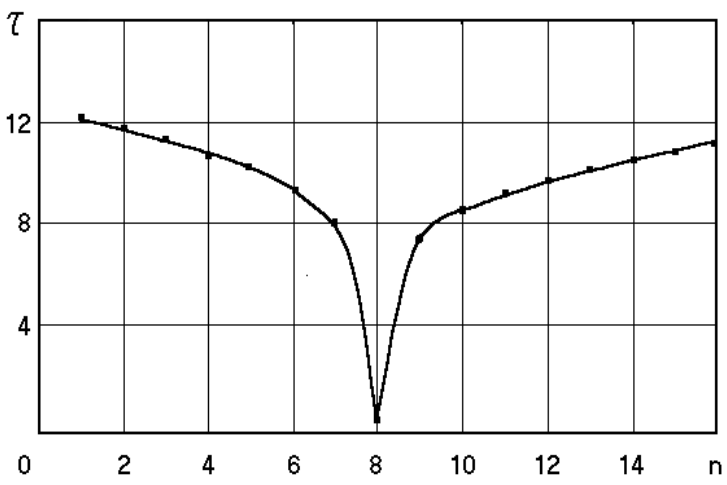

Fig.3. Relative computer time $\tau$ as the function of the curve number $n$.

The separating curve S-F has the minimal computer time among all of the trajectories. At the same time this curve can not be used as the basis for the time-optimal trajectory construction because the projection of the point $F$ to this curve is the same point $\mathrm{F}$, but the movement slows down near this point. Only the curves that lie under the curve S-F serve as the first part of the time-optimal trajectory with the following jump to the point $F$. The relative computer time $\tau$ of the optimal trajectories with acceleration effect (on the basis of curves 1-7, Fig. 2 ) is shown in Fig. 4 as the function of the curve number $n$. The curves 9-16 can be optimized too but in this case the time reduction about $10-15 \%$ only takes place. Fig. 4 shows that the total computer 
time increases when the start point approaches to the curve S-F, and on the contrary, the more acceleration can be obtained if the start point lies far from the curve S-F (from curve 7 to curve 1).

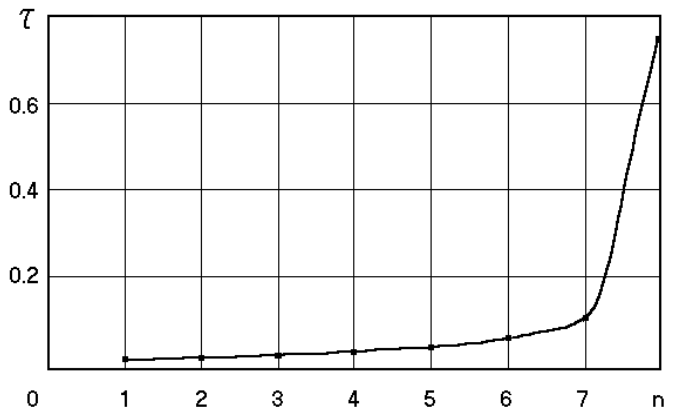

Fig.4. Relative computer time $\tau$ of the optimal trajectories with acceleration effect as the function of the curve number $n$.

So, the start point selection with at least one negative initial coordinate of the vector $X$ and the value of this coordinate that gives the start point position under the separating line are the sufficient conditions for the acceleration effect appearance.

More detail analysis shows that the negative value of the start point coordinate below the separate line is the sufficient condition for the acceleration effect but is not the necessary. The phase diagram of Fig. 5 includes two types of the separate lines. The first line AFB separates the trajectories that draw to the final point $\mathrm{F}$ from the left and from the right. The second separate line CTFB divides all the phase space to the two subspaces. All the points and trajectories that lie inside this separate line can not produce the acceleration effect. On the other hand, all the points that lie outside the separate line and corresponding trajectories produce the acceleration effect. These geometrical conditions are the necessary and sufficient to obtain the acceleration effect.

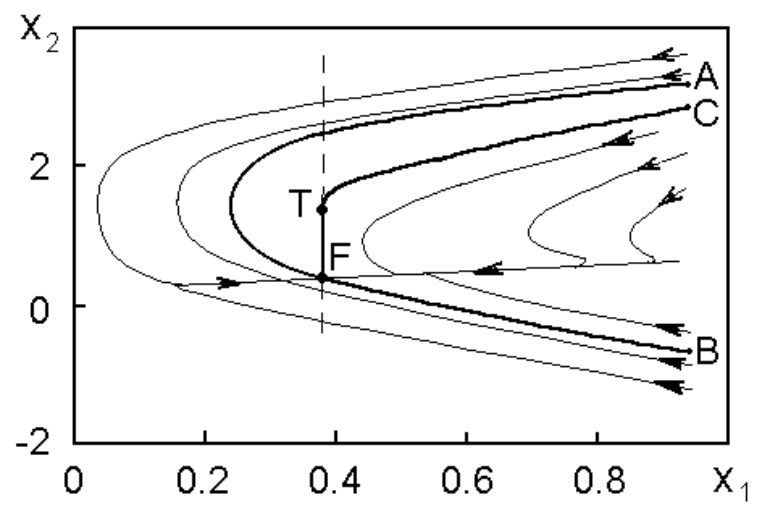

Fig.5. Phase diagram $x_{1}-x_{2}$ for one-node circuit.
The $\mathrm{N}$-dimensional case has been analyzed below. The second example corresponds to the circuit in Fig. 6.

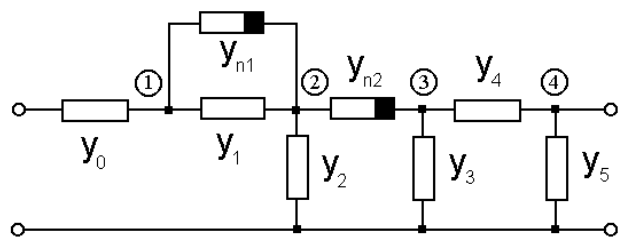

Fig.6. Four-node circuit topology.

This circuit has five independent variables $y_{1}, y_{2}, y_{3}, y_{4}, y_{5}(K=5)$ and four dependent variables $V_{1}, V_{2}, V_{3}, V_{4} \quad(M=4)$. Non-linear circuit elements can be determined by the following formulas: $y_{n 1}=a_{n 1}+b_{n 1} \cdot\left(V_{1}-V_{2}\right)^{2}, y_{n 2}=a_{n 2}+b_{n 2} \cdot\left(V_{3}-V_{2}\right)^{2}$. Non-linearity parameters $b_{n 1}, b_{n_{2}}$ are equal to 1.0. The state parameter vector $X$ includes nine components: $x_{1}^{2}=y_{1}, x_{2}^{2}=y_{2}, x_{3}^{2}=y_{3}, x_{4}^{2}=y_{4}, x_{5}^{2}=y_{5}$, $x_{6}=V_{1}, x_{7}=V_{2}, x_{8}=V_{3}, x_{9}=V_{4}$. The optimization process system consists of nine equations, and the circuit model consists of four.

The phase space of the total states parameters has nine dimensions. The separate lines are transformed to the separate hyper-surfaces in this case. The phase projections of the separate hyper-surfaces (separate line one and separate line two), which correspond to the phase plane $x_{5}-x_{9}$ are shown in Fig. 7.

The region outside the separate line 2 includes the points and the trajectories that can produce the acceleration effect. In this case, as for the first example, the separate line 2 or more general the separate hyper-surface 2 defines the necessary and sufficient conditions for the existence of acceleration effect.

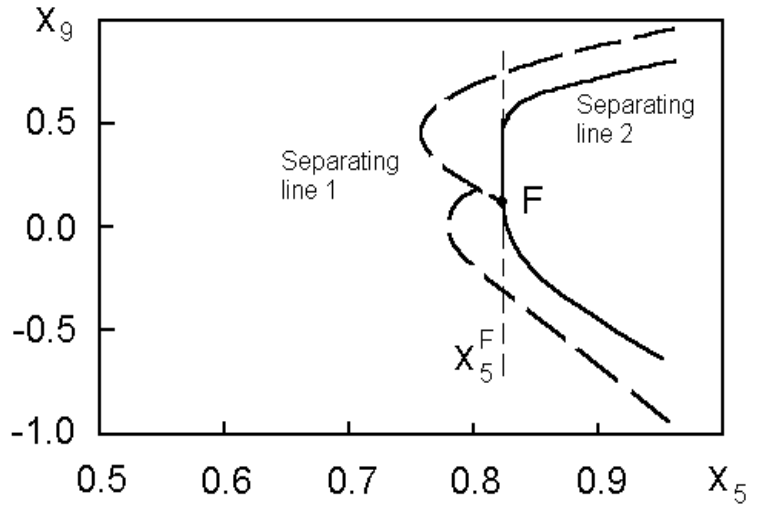

Fig.7. Phase diagram $x_{5}-x_{9}$ for four-node circuit. 
Active nonlinear circuits are analyzed below. A circuit of the transistor amplifier that consists of three transistor cells is shown in Fig. 8.

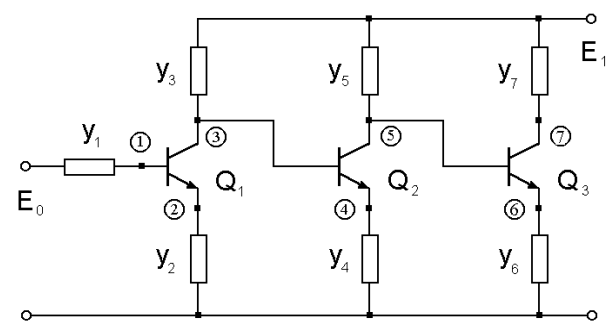

Fig.8. Three-cell transistor amplifier.

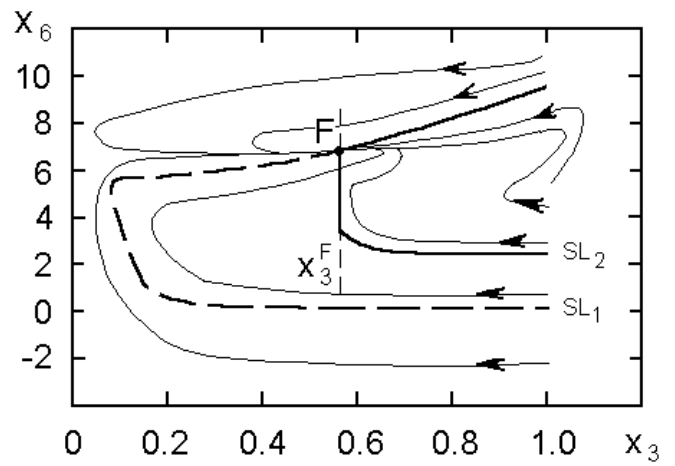

(a) $\quad x_{i}^{0}=1.0, i=1,2, \ldots, K(K=3)$

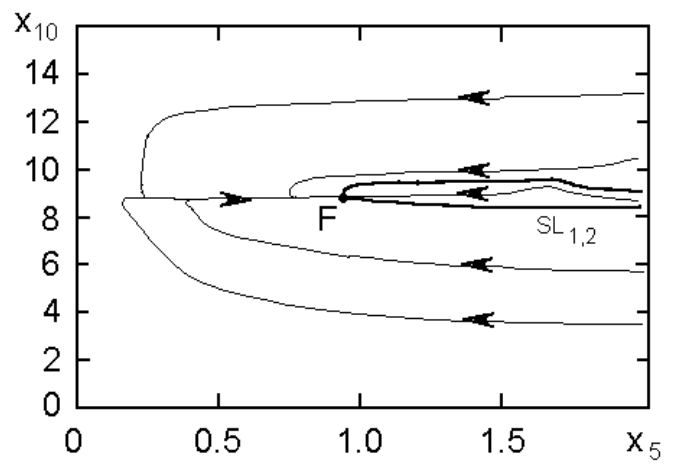

(b) $\quad x_{i}^{0}=2.0, i=1,2, \ldots, K(K=5)$.

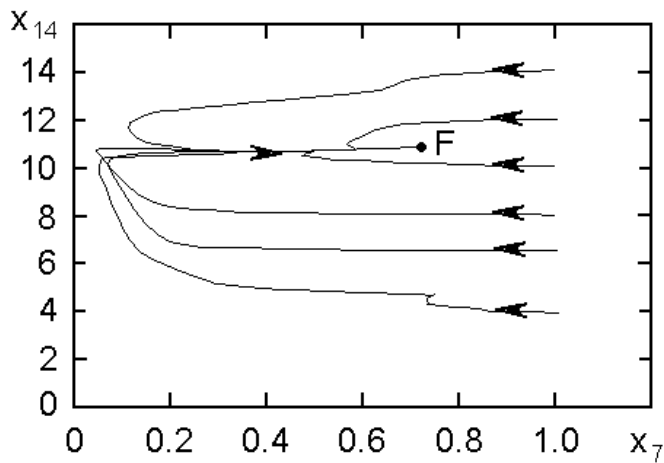

(e) $\quad x_{i}^{0}=1.0, i=1,2, \ldots, K(K=7)$.
The Ebers-Moll static transistor model is used [23]. The one, two and three transistor cell circuits were analyzed separately. The one transistor cell circuit was analyzed as the first example. In this case we have three independent variables $y_{1}, y_{2}, y_{3}$ as admittance $(K=3)$ and three dependent variables $V_{1}, V_{2}, V_{3}$ as nodal voltages $(M=3)$. The state parameter vector $X$ includes six components: $x_{1}^{2}=y_{1}, \quad x_{2}^{2}=y_{2}, \quad x_{3}^{2}=y_{3}, \quad x_{4}=V_{1}, \quad x_{5}=V_{2}$, $x_{6}=V_{3}$. Fig. 9 corresponds to the trajectory graphs of the modified traditional design strategy for three above mentioned types of the transistor amplifier.

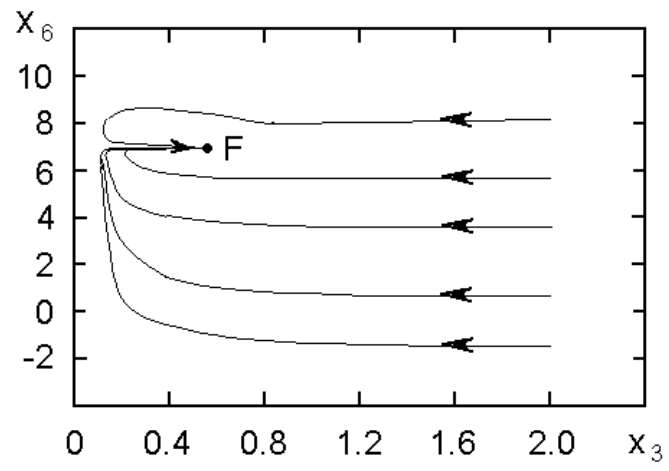

(c) $\quad x_{i}^{0}=2.0, i=1,2, \ldots, K(K=3)$.

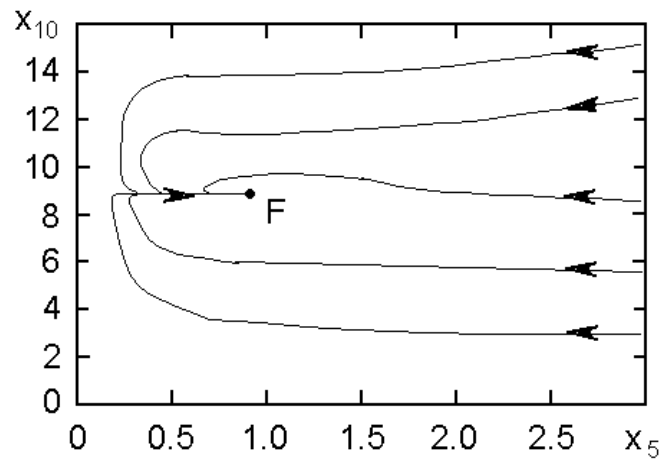

(d) $\quad x_{i}^{0}=3.0, i=1,2, \ldots, K(K=5)$.

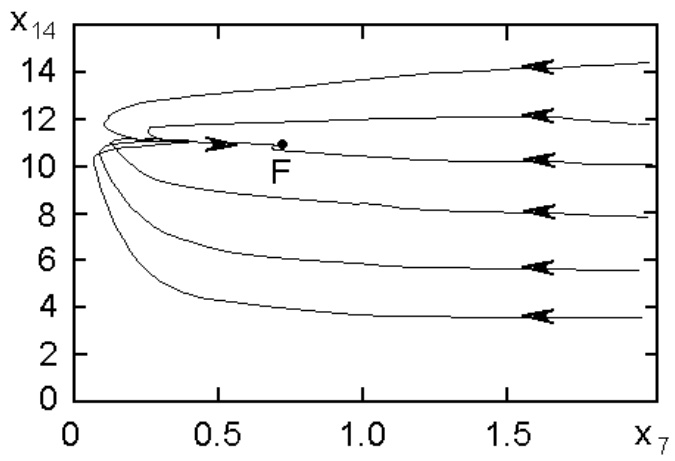

(f) $\quad x_{i}^{0}=2.0, i=1,2, \ldots, K(K=7)$.

Fig.9. Family of the curves that correspond to the MTDS and separate lines for: (a), (b) one-cell; (c), (d) twocell; and (e), (f) three-cell transistor amplifier. 
Fig. 9 (a), (b) shows the behavior of the trajectory projections in the plane $x_{3}-x_{6}$. Fig. 9 (a) corresponds to the initial coordinate values $x_{i}^{0}=1.0$, and Fig. 9 (b) to the values $x_{i}^{0}=2.0$ for $i=1,2,3$. There is a great difference between the active and the passive circuits. The separate lines 1 and 2 (the projections of the corresponding separate hyper surfaces) have a very strong configuration for $x_{i}^{0}=1.0$, which explains the presence or absence of the acceleration effect. On the contrary, the separate hyper surface projections disappear in the plane $x_{3}$ $x_{6}$ for the initial values $x_{i}^{0}=2.0$. This means that the acceleration effect is always observed at any value of the $x 6$ coordinate, because all trajectories include the possibility of a moving point jumping to the finish point. It is very interesting that the circuit complication bring to the further expansion of the acceleration effect region. We can see this property from Fig. 9 (c), (d) and (e), (f). Fig. 9 (c), (d) correspond to the two-cell transistor amplifier and Fig. 9 (e), (f) to the three cell amplifier. There is a significant reduction of the region of the acceleration effect absence for two cell amplifier, Fig. 9 (c). The projections of the separate hyper surface (separate lines 1 and 2) in the plane $x_{5}-x_{10}$ have the same behavior and very narrow region of the acceleration effect absence for $x_{i}^{0}=2.0$, $i=1,2,3,4,5$. The acceleration effect always exists for $x_{i}^{0}=3.0$ as we can see in Fig. 9 (d). The separate hypersurface disappears completely for the three cell transistor amplifier, as we can see in Fig. 9 (e), (f). We can implement the acceleration effect for circuit design for almost all starting points and for all trajectories.

\section{Conclusion}

The initial point selection permits obtain acceleration effect with a great probability. The trajectory analysis of various design strategies shows that the conception of the separate line or the separate hyper surface in general case is very helpful to understand and define the necessary and sufficient conditions for the design process acceleration effect existence. The separate hyper surface defines the start points and the trajectories that can produce the acceleration effect and can be used for the optimal design trajectory construction. The selection of the initial points outside of the separate hyper surface is the necessary and sufficient conditions for the acceleration effect existence.
The separate hyper surface has the complex structure in general case. However, the situation is simplified for the active nonlinear circuits because a disappearance of the separate hyper surface for more complicated circuits. It means that the acceleration effect can be realized always for the complex active circuits. This effect further reduces the total computation time and serves as a basis for finding and constructing an optimal or quasi-optimal algorithm.

\section{Acknowledgment}

This work was supported by the Autonomous University of Puebla, under project VIEP2021.

\section{References:}

[1] O. Osterby, and Z. Zlatev, Direct Methods for Sparse Matrices, Springer-Verlag, N.Y., 1983.

[2] A. George, On Block Elimination for Sparse Linear Systems, SIAM J. Numer. Anal., Vol.11, No.3, 1984, pp. 585-603.

[3] F.F. Wu, Solution of Large-Scale Networks by Tearing, IEEE Trans. Circuits Syst., Vol.CAS23, No.12, 1976, pp. 706-713.

[4] A. Sangiovanni-Vincentelli, L.K. Chen, and L.O. Chua, An Efficient Cluster Algorithm for Tearing Large-Scale Networks, IEEE Trans. Circuits Syst., Vol.CAS-24, No.12, 1977, pp. 709-717.

[5] R.K. Brayton, G.D. Hachtel, and A.L. Sangiovanni-Vincentelli, A survey of optimization techniques for integrated-circuit design, Proceedings IEEE, Vol.69, No.10, 1981, pp. 1334-1362.

[6] A.E. Ruehli (Ed.), Circuit analysis, simulation and design Part 2, Elsevier Science Publishers, Amsterdam, 1987.

[7] G. Stehr, M. Pronath, F. Schenkel, H. Graeb, and K. Antreich, Initial sizing of analog integrated circuits by centering within topology-given implicit specifications, Proc. of the IEEE/ACM Int. Conf. CAD, 2003, pp. 241246.

[8] M. Hershenson, S. Boyd, and T. Lee, Optimal design of a CMOS op-amp via geometric programming, IEEE Trans. CAD of Integrated Circuits and Systems, Vol.20, No.1, 2001, pp. $1-21$.

[9] D. Nam, Y. Seo, L. Park, C. Park, and B. Kim, Parameter optimization of an on-chip voltage reference circuit using evolutionary programming. IEEE Trans. Evolutionary Comp., Vol.5, 2001, pp. 414-421. 
[10] N.F. Paulino, J. Goes, and A. Steiger-Garcao, Design methodology for optimization of analog building blocks using genetic algorithms. Proc. Symposium Circuits and Systems, 2001, pp. 435-438.

[11] G. Alpaydin, S. Balkir, and G. Dundar, An evolutionary approach to automatic synthesis of high performance analog integrated circuits, IEEE Trans. Evolutionary Comp., Vol.7, 2003, pp. 240-252.

[12] A. Srivastava, T. Kachru, and D. Sylvester, Low-Power-Design Space Exploration Considering Process Variation Using Robust Optimization, IEEE Trans. CAD of Integrated Circuits, Vol.26, No.1, 2007, pp. 67-79.

[13] B. Liu, Y. Wang, Z. Yu, L. Liu, M. Li, Z. Wang, J. Lu, and F.V. Fernandez, Analog circuit optimization system based on hybrid evolutionary algorithms. Integration the VLSI J., Vol.42, No.2, 2009, pp. 137-148.

[14] F. Yengui, L. Labrak, F. Frantz, R. Daviot, N. Abouchi, and I. O'Connor, A hybrid GA-SQP algorithm for analog circuits sizing, circuits and systems, Circuits and Systems, Vol.3, 2012, pp. 146-152.

[15] M.R.M. Ridzuan, E.E. Hassan, A.R. Abdullah, N. Bahaman, and A.F.A. Kadir, A new meta heuristic evolutionary programming (NMEP) in optimizing economic energy dispatch, $J$. Telecomm. Electron. Comp. Engineer., Vol.8, No.2, 2016, pp. 35-40.

[16] J. Robinson, and Y. Rahmat-Samii, Particle swarm optimization in electromagnetic. IEEE Trans. Antennas and Propagation, Vol.52, No.2, 2004, pp. 397-407.
[17] M.A. Zaman, M. Gaffar, M.M. Alam, S.A. Mamun, and M.A. Matin, Synthesis of antenna arrays using artificial bee colony optimization algorithm, Int. J. of Micro. and Optic. Technol., Vol.6, No.8, 2011, pp. 234-241.

[18] I.S. Kashirskiy, Y.K. Trokhimenko, General optimization for electronic circuits, Tekhnika, Kiev, 1979.

[19] V. Rizzoli, A. Costanzo, and C. Cecchetti, Numerical optimization of broadband nonlinear microwave circuits, Proceedings of IEEE MTT-S Int. Symposium, Vol.1, 1990, pp. 335-338.

[20] E.S. Ochotta, R.A. Rutenbar, and L.R. Carley, Synthesis of high-performance analog circuits in ASTRX/OBLX, IEEE Trans. CAD Integrated Circuits and Systems, Vol.15, No.3, 1996, pp. 273-294.

[21] A. Zemliak, Analog circuit optimization on basis of control theory approach, COMPEL: The Int. J. Computation and Mathematics in Electrical and Electronic Engineering, Vol.33, No.6, 2014, pp. 2180-2204.

[22] A. Zemliak, F. Reyes, and S. Vergara, Study of different optimization strategies for analogue circuits, COMPEL: The Int. J. Computation and Mathematics in Electrical and Electronic Engineering, Vol.35, No.3, 2016, pp. 927-942.

[23] G. Massobrio, and P. Antognetti, Semiconductor Device Modeling with SPICE, Mc. Graw-Hill, Inc., N.Y., 1993.

\section{Creative Commons Attribution License 4.0 (Attribution 4.0 International, CC BY 4.0)}

This article is published under the terms of the Creative Commons Attribution License 4.0 https://creativecommons.org/licenses/by/4.0/deed.en_US 